\title{
TRAINING AND LEARNING MINIMALLY INVASIVE UROLOGY BANGLADESH PERSPECTIVES
}

\author{
MA SALAM
}

Chairman and Professor of Uro-oncology, Urology and Transplantation Foundation of Bangladesh, 57/9 Panthapath, Dhaka 1205, Bangladesh

\section{Introduction}

World wide the demand for minimally invasive specialty surgical care has exploded, and our healthcare system is also being stressed to capacity. Currently the need for highly trained, skilled surgical professionals has never been more critical. Surgeons today need to acquire knowledge and skills that reach beyond the classic teaching models of surgical education, and cover such variety of advanced skill and knowledge on laparoscopic, endoscopic and robotic technology for perfect leadership in health care management.

The century-old teaching paradigms of "see one, do one, teach one" and training by opportunity are inappropriate in a surgical world of rapidly introduced advanced technologies. Surgical simulation offers extraordinary opportunities to teach multiple clinical scenarios in a safe, nonhuman patient environment where performance feedback is immediate and objective.

The landscape of surgery is constantly changing. Worldwide, new standards and criteria have been produced for accreditation of health care facilities used for training $[1,2]$. The existing curriculum for each of the specialties has been remodeled to a competence- based format in line with the abilities required to undertake the essential roles of a surgeon[3].

Currently in most of the advanced medical institutions have introduced new basic surgical skills education and training course with simulation. Trainees' progress is assessed by simulation based assessment and formal examinations and the simulators are included in the exit examination.

There is a strong demand for more clinical accountability and the application of innovative technology to the surgical training program[3]. Each of these factors requires significant revisions to our current approaches to surgical training in Bangladesh.

Correspondences: Muhammad Abdus Salam, Chairman and Professor of Uro-oncology, Urology and Transplantation Foundation of Bangladesh, 57/9 Panthapath, Dhaka 1205, Bangladesh, E-mail: masalamurology@yahoo.com
Training for advanced expertise in surgical care The current trend in surgery toward minimally invasive and non-invasive therapeutic procedures is another field of newer technologies[6,7]. This trend represents a switch from direct, hands-on surgical approaches to indirect, "hands-off" approaches (e.g. laparoscopic, catheter-based, robot-aided, and computer-aided procedures). This switch coincides with moves by surgeons from unimodal therapy (e.g., resection and reconstruction) to multimodal therapy (e.g. biologically tagged, image-guided, and dexterity-enhanced procedures).

The single clearest statement of the general roles required of the medical training in general, is that made by the Can MEDS 2000 project[5], now being incorporated into each residency program in many developed countries. The Can MEDS framework provides a useful structure for reviewing the changes that any training program must undertake if it is to provide relevant training.

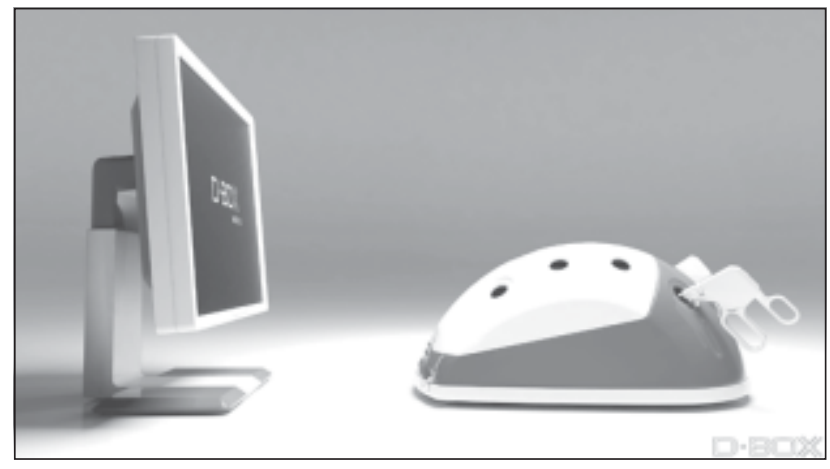

Fig.-1: A innovative laparoscopic training solution. $D$ box Basic simulator is designed to exercise and test the skills of surgeons ability. It evolved from the idea of making an affordable, suitable, portable and practical training arena for laparoscopy.

Therefore, the basic clinical training (BCT) in Bangladesh should evolve from a general year to provide essential grounding in medical skills and knowledge relevant to the various branches of surgery. More elective time within the years 2 to 5 will also need to be available for rotations, particularly for those interested in research, and basic science studies $[8,9,10]$. 


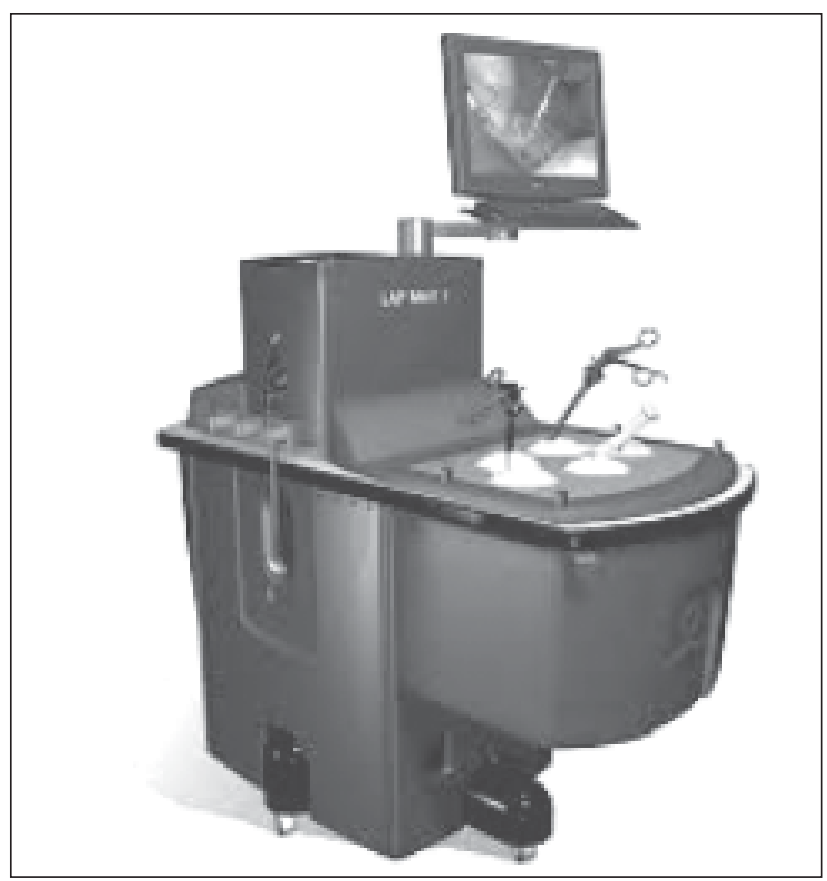

Fig.-2: Medical simulators which includes URO Mentor, GI Mentor, and PERC Mentor, allows the practice of laparoscopic procedures and basic laparoscopic skills.

The minimally invasive surgery is challenging requiring significant allocation of resident and faculty time, equipments and appropriate funds for consistent training results. Recent advances in virtual reality technology have demonstrated its potential for enhancing surgical skills training, and many virtual reality systems are now commercially available. Virtual reality provides the opportunity for very detailed feedback and may allow for more critical measurement of trainee performance than is possible in the real world.

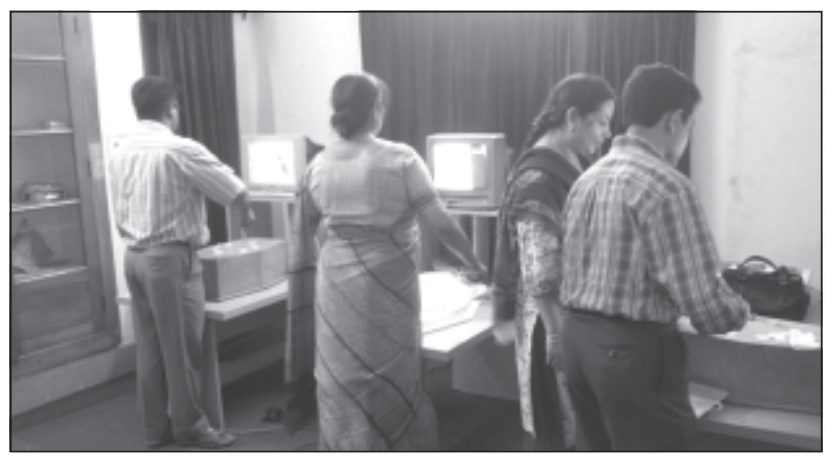

Fig.3: A small laparoscopic training center of Urology and Transplantation Foundation of Bangladesh (UTFB) for learning laparoscopic surgery. The center can take five students at a time and it operates a 5 days compact and comprehensive course.

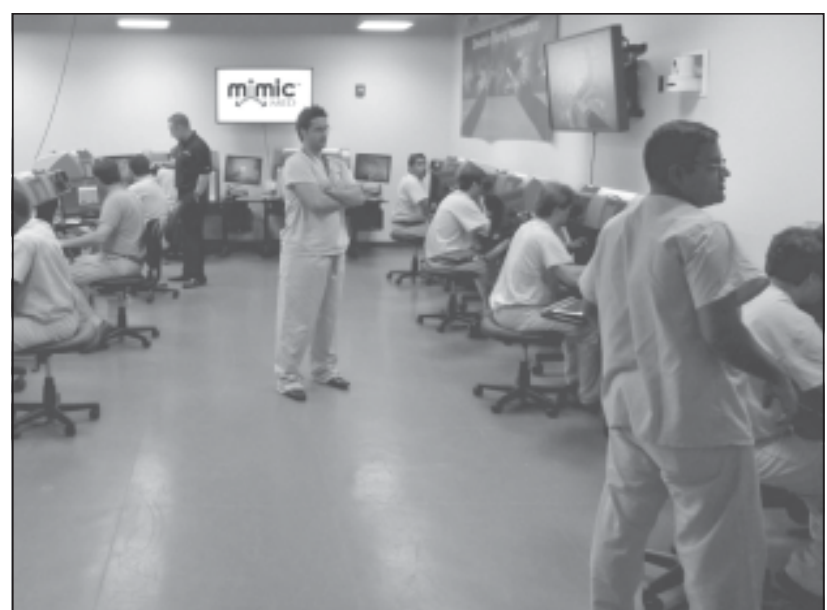

Fig.-4: Robotic Simulation Training at the upcoming Clinical Robotic Surgery Association 5th Worldwide Congress on October 3-5, 2013 in Washington, D.C. These courses are designed to give the user a basic understanding of the controls of the robotic system; to introduce users to virtual reality as a training tool; and to demonstrate the latest advances in robotic training simulation.

Measures of precision and accuracy as well as error rates can be calculated easily. Two prospective trials have demonstrated that residents who have been trained on low fidelity (not very life like) virtual reality models (laparoscopic box trainers) make fewer intra-operative errors when performing a laparoscopic cholecystectomy than do residents who have not had the benefit of simulation training[11,12].

Surgical simulation and simulated surgery using models and virtual reality (VR) has seen the most rapid growth of development in the last decade. Practicing suture knot-tying and suturing fake (phantom) tissues like a chicken breast or a silicone slab of "tissue" has been the criterion-standard for open surgery fundamental skill simulation. Because complete open surgeries are difficult to model or simulate, only with the advent of minimally invasive surgery (MIS), laparoscopy, did innovators begin developing virtual reality simulators modeling the video images seen by surgeons doing laparoscopy.

Reality-based simulators are ones in which the learner interacts with solid, real-life materials and uses surgical instruments expected to be used in the operating room.

The simulator is designed to practice skills of transurethral resection of the prostate. The simulator is designed to work out of possession of the endosurgical tools, practical skills and techniques of endourologycal interventions. 


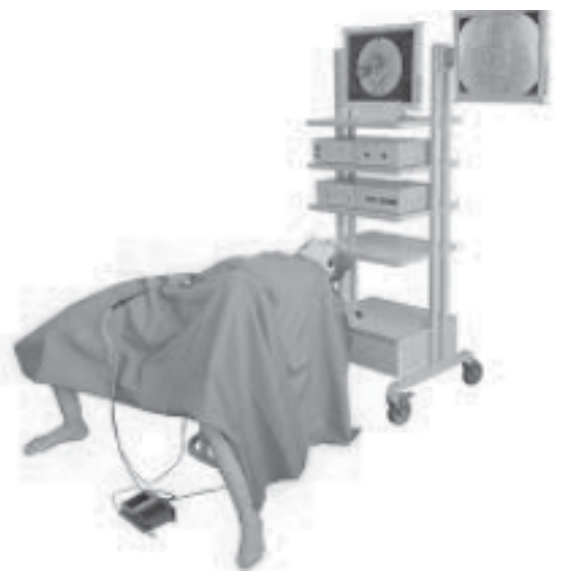

Fig.-5: Virtual simulator Endourology

Web-based simulation allows learners to interact with online curricula, exercising the cognitive aspects of surgery (e.g., what are the steps of a gallbladder removal, what surgical instruments are needed). The most technologically sophisticated class of simulator is the VR simulator, which features a computer-generated model of anatomy and requires that the learner use near similar instrumentation to interact with virtual tissue structures and models.

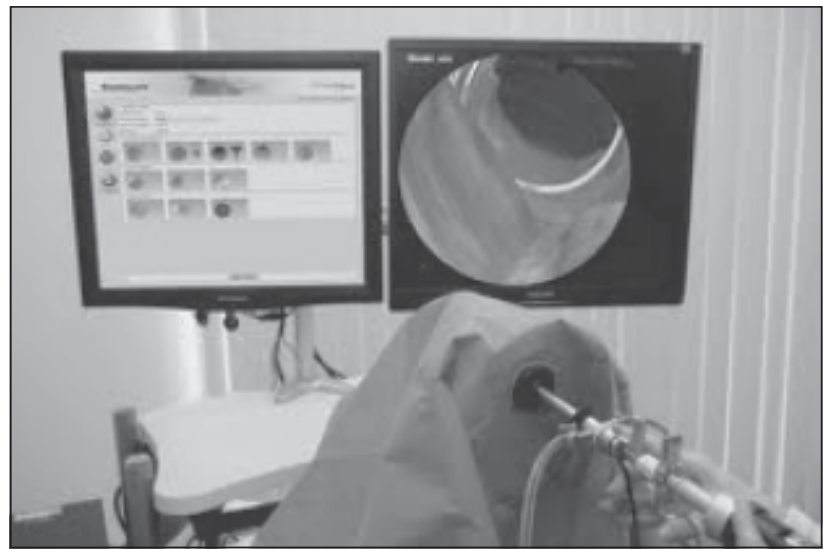

Fig.-6: The surgical simulator for TURP. TURPSim is state-of-the-art training tools for endoscopic surgery. Trans-Urethral Resection of the Prostate (TURP) is currently the most effective treatment of Benign Prostate Hyperplasia (BPH). With a wide range of prostate anatomies, pathologies and operative complications, this technique requires skills training and experience to achieve optimal results.

The accelerated growth of computer technology should be credited for many dramatic changes in medicine, such as advances in medical imaging technology (ultrasound, CT and MRI scanning). Computer based digital imaging technology has become an integral part of surgical therapy through its integration at all levels of surgical care.

Departure from traditional two- dimensional (2D) imaging (i.e., radiographs) to the $3 \mathrm{D}$ visualizations available through such technologies as CT scanning and virtual reality has offered effective new tools for preoperative diagnostics and planning, intra-operative navigation, and robotic surgery.

Philosophy of training today, means finding flexible ways for the trainee to master these new diagnostic and therapeutic technological advances and to acquire the core competencies of their subspecialty.

It has been observed and confirmed currently using the operating room for basic skill acquisition may be inefficient and expensive, especially for laparoscopic operations. This study determines if laparoscopic skills training using simulated tasks on a video-trainer improves the operative performance of surgery residents[21].

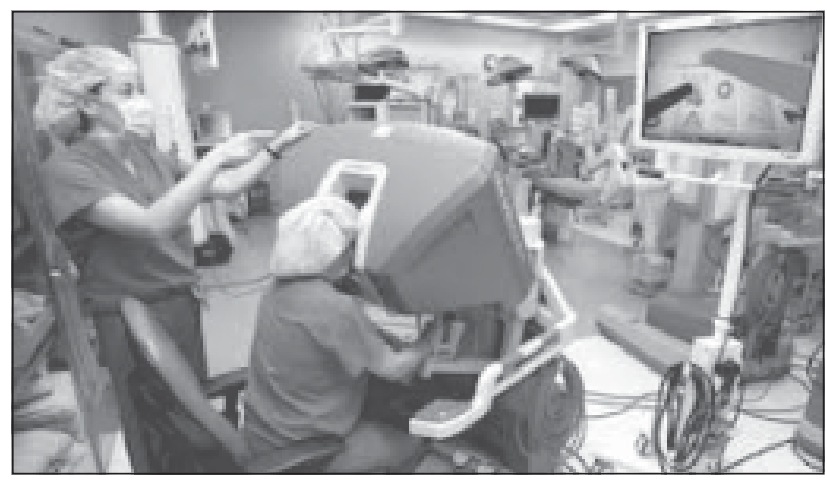

Fig.-7: Da Vinci Si surgical system. gains hands-on experience using the da Vinci Robotic System.

Second- and third-year residents $(n=27)$ were prospectively randomized to receive formal laparoscopic skills training or to a control group. At baseline, residents had a validated global assessment of their ability to perform a laparoscopic cholecystectomy based on direct observation by three evaluators who were blinded to the residents' randomization status. Residents were also tested on five standardized video-trainer tasks. The training group practiced the video-trainer tasks as a group for 30 minutes daily for 10 days. The control group received no formal training. All residents repeated the video-trainer test and underwent a second global assessment by the same three blinded evaluators at the end of the 1-month rotation. Within-person improvement was determined; improvement was adjusted for differences in baseline performance. 
Five residents were unable to participate because of scheduling problems; 9 residents in the training group and 13 residents in the control group completed the study. Baseline laparoscopic experience, video-trainer scores, and global assessments were not significantly different between the two groups. The training group on average practiced the video-trainer tasks 138 times (range 94 to 171 times); the control group did not practice any task. The trained group achieved significantly greater adjusted improvement in video-trainer scores (five of five tasks) and global assessments (four of eight criteria) over the course of the four-week curriculum, compared with controls[21].

This become evident that supervised perfect training improves video-eye-hand skills and translates into improved operative performance for junior surgery residents. Surgical curriculum should contain structured training program of minimally invasive urology before they are involved in surgical interventation[21].

\section{Role of Faculty to transfer skills of MIU(Minimally Invasive Urology)}

Training program must ensure that residents are experienced in efficient clinic- and team-based treatment models. Emphasis on the trainee -trainer ratio expected to be $1: 1$ to $1: 5$, as recommended by most training program, to ensure responsibility and accountability for training. Training units should be adequately funded and regularly assessed and the outstanding trainer should be awarded to encourage the teaching and training environment. We have to encourage the transfer of knowledge to the future surgeons and to promote the culture of training $[13,14,15]$.

\section{Enhancing the communicating skill of MIU(Minimally Invasive Urology)}

Generally the medical professionals are weak in communicating facts to their patients and their nearest and dearest. Communication skills are very much essential to the surgical practice but, as with many other fundamentals, are neither taught nor evaluated systematically during the academic program ${ }^{16}$. Evaluations of the communication skills identify, for instance, whether a resident can clearly and empathically convey to patients their diagnosis and treatment options; or clearly ask a question to a colleague when seeking a consultation; or write clear, concise progress notes; or dictate a focused, relevant consultation letter with clear treatment options[17].
Therefore, communication skill courses should be integrated to the training program.

\section{Evaluation of competency of of MIU(Minimally Invasive Urology)}

Surgical training evaluations are almost entirely based on rotation evaluation forms that program are redesigning to reflect the training programs competencies discussed above. Unfortunately, rotation evaluations are notoriously unreliable. More accurate assessment of trainee competency will depend on clear description of the particular skills being assessed; on use of more standardized tools, such as Objective Structured Clinical Examinations (OSCES), learning modules, and written examinations; and on comprehensive evaluations involving not just the immediate supervisor but also team members, patients, fellow residents, and other surgeons[18].

More comprehensive assessments can be done through Web- based evaluation software, already used in some medical programs. Such evaluation will provide more timely and anonymous feedback to the trainee and will be more accurate than the now-common singlesupervisor evaluation. In addition, competency will need to be linked to experience. Although repetition may not guarantee competence, lack of repetition all but ensures incompetence[19].

Advanced Training in other disciplines using simulators Simulators are in constant use in Army and civil aviation training institute like any other discipline. A flight simulator is a device that artificially re-creates aircraft flight and the environment in which it flies, for pilot training, design, or other purposes. It includes replicating the equations that govern how aircraft fly, how they react to applications of flight controls, the effects of other aircraft systems, and how the aircraft reacts to external factors such as air density, turbulence, wind shear, cloud, precipitation, etc.

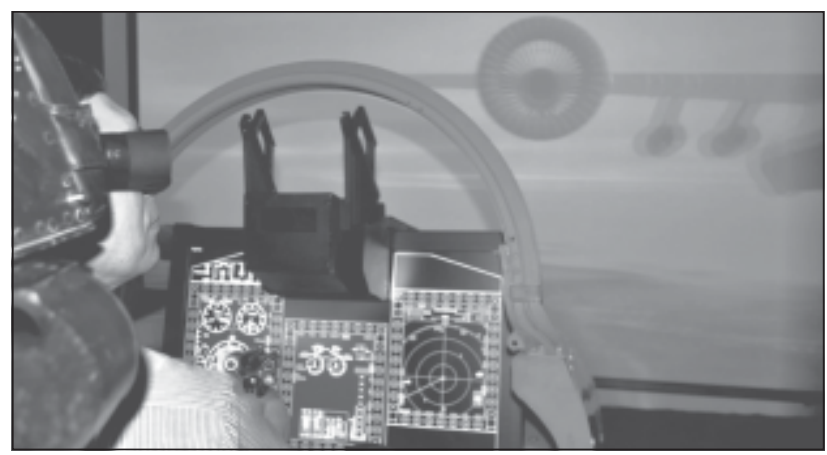

Fig.-8: Virtual reality has been adopted by the military. MiG is claiming a major first in military aviation with the launch of a 3D flight simulator at the Dubai Air Show. 


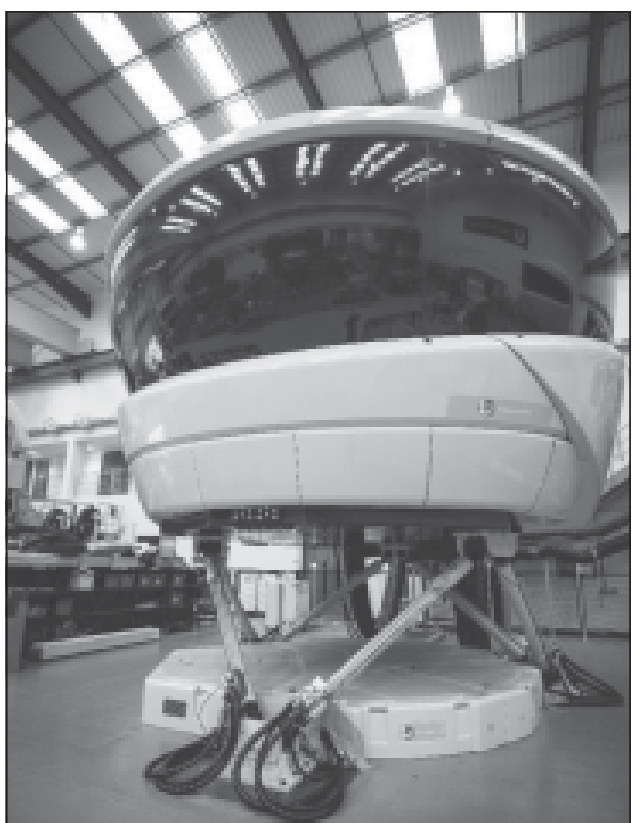

Fig.-9: The brand new Boeing 777 full-flight simulator (FFS) is being manufactured by L-3 Link Simulation \&Training UK (L-3 Link UK) at its Crawley, UK facility.

Flight simulation is used for a variety of reasons, including flight training, mainly for pilots, the design and development of the aircraft itself, and research into aircraft characteristics and control handling qualities[20]. The highest level of flight simulator for training Commercial Air Transport (CAT) pilots is known as a Full Flight Simulator (FFS), and for training military pilots the highest level is a Full Mission Simulator (FMS). Civil aviation and military use of simulator.

\section{Conclusion}

Knowledge is the most powerful tool of the surgeons. The surgical training program should strive to foster an environment of learning and research (basic and clinical), which enables our graduates to critically appraise surgical literature and keep abreast of surgical advances years after graduating.

As we move into the future, one of the many challenges of surgical education will be the need to obtain training in areas not traditionally covered in medical school or many hospital training programs. These areas consist of leadership development and associated non clinical skills.

External Mandates already have irrevocably changed the surgical training all over the world. How do we stand prepared to address the increasingly stringent credentialing for new minimally access and minimally invasive surgical procedures, and evidence based medicine surgical practice? Therefore, surgical trainees today face the challenge of not only becoming proficient clinically but also acquiring a new knowledge of new techniques and developing fundamental skills of leadership, advocacy, and policymaking in order to become effective surgical leaders for tomorrow.

Although MIS techniques, laparoscopic and robotic, are ideally suited for computer-assisted or VR training, medical decision-making simulation for MIS and open surgery is still in its infancy and is arguably the most important aspect of effective surgical practice. Few reality-based and most VR-based simulators allow for real-time objective performance tracking, making the VR simulation platforms ideally suited for surgical education.

We need to establish a centre in all medical colleges and tertiary institute to share the knowledge of the advanced laparoscopic work performed, and its work and reputation progressed we would like to establish a centre of excellence laparoscopic institute there is an ample opportunity to learn and do all practical minimally invasive surgeries in one of the most modern laparoscopic wet lab, Cadaveric dissection to offer a live and realistic understanding of tissue plane and critical anatomy which is required for all laparoscopic and endoscopic trainees of all branches of surgical disciplines.

\section{Conflict of Interest : None Declared}

\section{References}

1. Collins, John P. New standards and criteria for accreditation of hospitals and posts for surgical training... ANZ Journal of Surgery. 78(4):277-281, April 2008.

2. Karle, Hans's .Global Standards and Accreditation in Medical Education: A View from the WFME. Academic Medicine. Impact of International Medical Graduates on U.S. and Global Health Care: Proceedings of the 50th Anniversary Invitational Conference of the Educational Commission for Foreign Medical Graduates. 81(12) Supplement: S43-S48, December 2006.

3. Pandey, V. A. 1; Wolfe, J. H. N. 1; Liapis, C. D. 2; Bergqvist, D. 3; The European Board of Vascular Surgery The examination assessment of technical competence in vascular surgery. British Journal of Surgery. 93(9):1132-1138, September 2006

4. Moore JW. Education versus training. J. Chem. Educ. 1998; 75: 2. 
5. Societal Needs Working Group. Can MEDS 2000 project. Skills for the new millennium. Annals of the Royal College of Physicians and Surgeons of Canada 1996; 29:206-16

6. Halsted WS. rtiz-Oshiro, Elena MD, PhD ; Martinez, Cristina Pardo MD, PhD ; Ramirez, Joaquin Gomez MD ; Gonzalez Lopez, Pablo A. MD +; Perez, Cristina Fernandez MD, PhD ++; Carmona, Jose Angel De Diego MD, PhD ; Fernandez-Represa, Jesus Alvarez MD, PhD Lessons Learned From Long-term University Training in Minimally Invasive Surgery in Spain. Surgical Laparoscopy, Endoscopy \& Percutaneous Techniques. 18(6):583-588, December 2008.

7. Birch, Daniel W; Misra, Monali; Farrokhyar, Forough. The feasibility of introducing advanced minimally invasive surgery into surgical practice. Canadian Journal of Surgery. 50(4):256-260, August 2007.

9. The training of the surgeon. Bull. Johns Hopkins Hosp. 1904; 15: 267-75 Cobb RA, Baigrie RJ, Harris P, Harries PG, Shaper K, FoxA, and others. What constitutes general surgical training? Evidence from the log books of trainees in one district general hospital. Ann R Coll Surg Engl 1994;76(3 Suppl):117-2

10. McColl I. The Guy's surgical training programme: a report on the first five years. Br. J. Surg. 1977; 64: 745-6.

11. Gurusamy, K. 1; Aggarwal, R. 2; Palanivelu, L. 3; Davidson, B. R. 1 Systematic review of randomized controlled trials on the effectiveness of virtual reality training for laparoscopic surgery. British Journal of Surgery. 95(9):1088-1097, September 2008

12. Seymour, Neal E; Gallagher, Anthony G.; Roman, Sanziana A; O'Brien, Michael; Bansal, Vipin K; Andersen, Dana K; Satava, Richard M. Virtual Reality Training Improves Operating Room Performance: Results of a Randomized, DoubleBlinded Study. Annals of Surgery. 236(4):458-464, October 2002

13. MacFarlane, John K. MD, FRCSC the Surgeon as Professional: A Challenge to Our Educators.
Archives of Surgery. Pacific Coast Surgical Association. 136(8):860-863, August 2001

14. Davidson, Patricia M.The Surgeon for the future and implications for training. ANZ Journal of Surgery. 72(11):822- 828, November 2002

15. Hargreaves $\mathrm{DH}$. A training culture in surgery. $\mathrm{Br}$. Med. J. 1996; 313: 1635-9

16. DesCoteaux JG, Wallis MV. Applying theory to design of communication-skills teaching at postgraduate medical level. Annals RCPSC 1998; 31:270-4.

17. Williams, B. C. 1; Hall, K. E. 1; Fitzgerald, J. T; Supiano, M. A. Use of standardized patients to teach functional assessment and communication skills to surgical and medical subspecialty house officers. Journal of General Internal Medicine. 19 Supplement 1:232-233, April 2004.

18. Suliburk, James W; Kao, Lillian S; Kozar, Rosemary A; Mercer, David W. Training Future Surgical Scientists: Realities and Recommendations. Annals of Surgery. 247(5):741749, May 2008

19. Bradley, Ciaran T; Brasel, Karen J, MPH Core Competencies in Palliative Care for Surgeons: Interpersonal and Communication Skills. American Journal of Hospice \& Palliative Medicine. 24(6):499507, December/January 2007.

20. Federal Aviation Administration (25 April 2013). "FAR 121 Subpart N-Training Program". Retrieved 28 April 2013.

21. Scott $\mathrm{DJ}^{1}$, Bergen PC, Rege RV, Laycock R, Tesfay ST, Valentine RJ, Euhus DM, Jeyarajah DR, Thompson WM, Jones DB. Laparoscopic training on bench models: better and more cost effective than operating room experience? J Am Coll Surg. 2000 Sep;191(3):272-83.

\section{Abbreviations:}

BCT : Basic Clinical Training

MIS : Minimally invasive Surgery

VR : Virtual Reality 